\title{
Hepatitis inespecífica asociada a otra Patología
}

\author{
Nonspecific hepatitis
}

BERRIOS REITERER Jorge ${ }^{1}$, CABELLO José ${ }^{2}$, COMBE Juan ${ }^{2}$ y BUSSALLEU Alejandro $^{3}$

${ }^{1}$ Profesor Principal de Medicina de la Universidad Peruana Cayetano Heredia, Jefe del Servicio de Gastroenterología del Hospital Cayetano Heredia.

${ }^{2}$ Médicos del Servicio de Gastroenterología del Hospital Cayetano Heredia.

${ }^{3}$ Profesor Auxiliar de Medicina de la Universidad Peruana Cayetano Heredia. Asistente del Servicio de Gastroenterología del Hospital Cayetano Heredia.

\section{SUMMARY}

In medical practice extrahepatic pathology is frequently seen associated to hepatitis. It has been usually denominated nonspecific reactive hepatitis, although it is not accepted by all. We report on 20 patients attended at Cayetano Heredia Hospital, pointing out the clinical, laboratory and histopathological findings.In this group the clinical manifestations of the primary disease are always predominant and at some time they develop fever, coluria, jaundice and a mild, painful hepatomegaly.

In the laboratory tests a conjugated hyperbilirrubinemia not exceeding 3 to 4 $\mathrm{mg} / \mathrm{dl}$, transaminases usually no more than 250 to 300 units and alkaline phosphatase in 2 to 3 times the normal values, are shown.

The liver biopsy performed in all cases show unspecific inflammatory changes.

We propose that the alterations are due to hepatic susceptibility to different possible factors, as: toxic, metabolic, circulatory, immunologic and some others yet unknown. The prognosis and treatment are related to the basic disease, evoiding the use of hepatotoxic drugs.

KEY WORDS: reactive hepatitis, nonspecific hepatitis.

\section{RESUMEN}

En la práctica médica es frecuente encontrar patología extrahepática a la que se asocian manifestaciones de hepatitis que ha recibido diferentes denominaciones, siendo la más usual hepatitis reactiva o reaccional inespecífica, que no obstante no es aceptada por todos. Como ejemplo se presentan 20 casos atendidos en el Hospital Cayetano Heredia, señalándose los hallazgos clínicos, de laboratorio y anátomo-patológicos.

Se establece que en el cuadro clínico predomina la enfermedad de fondo, presentándose en algún momeno fiebre, coluria, ictericia y hepatomegalia ligeramente dolorosa. Se encuentra hiperbilirrubinemia que no excede de 3 a $4 \mathrm{mg} / \mathrm{dl}$ a predominio de la conjugada; elevación de las transaminasas que no suelen pasar de 250-300 UI/dl y 
fosfatasa alcalina elevada en 2 a 3 veces lo normal. La biopsia hepática realizada en todos los casos está caracterizada por cambios inflamatorios inespecíficos: injuria o necrosis focal leve, infiltración celular de tipo portal y en alguna dilatación sinusoidal o cambios mínimos a nivel de los colangiolos.

Se plantea que las alteraciones se producen por la susceptibilidad hepática a un conjunto de posibles factores tóxicos, metabólicos, circulatorios, inmunológicos y otros aún no conocidos.

El pronóstico y tratamiento se relacionan con la enfermedad de fondo, evitando los hepatotóxicos.

PALABRAS CLAVE: hepatitis reactiva, hepatitis inespecífica reaccional.

\section{INTRODUCCION}

En el trabajo médico no es infrecuente asistir a pacientes que durante la evolución de una enfermedad extrahepática presentan en algún momento manifestaciones de compromiso del hígado, sin evidencias de localización en este órgano del causante del proceso inicial.

En estos casos en la histología hepática existen lesiones inflamatorias que han sido muy bien descritas en los libros de Anatomía Patológica; sin embargo en los textos de Medicina y Hepatología hay muy pocos datos sobre su descripción clínica, en tanto que en las publicaciones sobre la nomenclatura y clasificación de las enfermedades hepáticas ni se la menciona (1).

Se realiza el presente trabajo con el objeto de contribuir al conocimiento de tal situación, señalando el cuadro clínico, los hallazgos de laboratorio, su correlación con la anatomía patológica y un esbozo de la patogenia, estableciendo su pronóstico y tratamiento.

\section{MATERIAL Y METODOS}

Se consideran veinte pacientes que fueron internados en el Hospital Cayetano Heredia por enfermedades no hepáticas y que durante su hospitalización presentaron como complicación una alteración de grado variable en las pruebas de función hepática (PFH).

Se señalan los diagnósticos iniciales, destacando luego los síntomas y signos pertinentes a la complicación.

Se consignan los resultados de los exámenes de laboratorio, habiéndose efectuado en todos ellos dosajes de bilirrubina total y fraccionada, transaminasas glutámico oxalacética y glutámico pirúvica, fosfatasa alcalina, además de los exámenes pertinentes para cada caso.

Se precisan las conclusiones de la biopsia hepática realizada en todos los enfermos.

Se analizan y discuten los hallazgos. 


\section{RESULTADOS}

Se encuentran 15 pacientes de sexo femenino y 5 de sexo masculino de edad muy variable, más frecuente en las tres primeras décadas, pero aún presente en la década de los 71 a 80 años (Tabla $\left.N^{\circ} 1\right)$.

\begin{tabular}{|c|c|}
\hline \multicolumn{2}{|c|}{$\begin{array}{c}\text { TABLA N }^{2} 1 . \\
\text { HEPATITIS INESPECIFICA: } \\
\text { DISTRIBUCION DE LOS CASOS SEGUN SEXO Y EDAD. }\end{array}$} \\
\hline & SEXO \\
\hline $\begin{array}{l}\text { Femenino } \\
\text { Masculino } \\
\text { TOTAL }\end{array}$ & $\begin{array}{l}=15(75 \%) \\
=5(25 \%) \\
=\quad 20(100 \%)\end{array}$ \\
\hline \multicolumn{2}{|r|}{ EDAD } \\
\hline $\begin{array}{r}0-10 \\
11-20 \\
21-30 \\
31-40 \\
41-50 \\
51-60 \\
61-70 \\
71-80 \\
\text { TOTAL }\end{array}$ & $\begin{array}{l}=4(20 \%) \\
=4(20 \%) \\
=4(20 \%) \\
=0(0 \%) \\
=1(5 \%) \\
=2(10 \%) \\
=4(20 \%) \\
=1(5 \%) \\
=20(100 \%)\end{array}$ \\
\hline
\end{tabular}

Los hallazgos histológicos corresponden a los de hepatitis inespecífica en los 20 pacientes.

Los diagnósticos asociados son: infección urinaria, colecistitis calculosa, fiebre de causa indeterminada, obstrucción intestinal, absceso cerebral, gastritis erosiva, hemorragia digestiva alta, absceso apendicular residual, bronconeumonia y tuberculosis pulmonar. La frecuencia de estos diagnósticos y las características bioquímicas de la hepatitis inespecífica se precisa en la Tabla $\mathrm{N}^{\mathrm{o}} 2$.

\begin{tabular}{|c|c|c|c|c|c|}
\hline \multicolumn{6}{|c|}{$\begin{array}{l}\text { TABLA Ne2. } \\
\text { HEPATITIS INESPECIFICA: RESULTADO DE LAS } \\
\text { PRUEBAS DE FUNCION HEPATICA SEGUN } \\
\text { DIAGNOSTICO. }\end{array}$} \\
\hline Diagnóstico & B. Tot & B. Con & SGO & SGP & F.Alc \\
\hline Infección Urinaria & 1.4 & 0.8 & 14 & 37 & $\mathrm{~N}$ \\
\hline Infección Urinaria & 3.5 & 2.8 & 30 & 150 & $2 \mathrm{~N}$ \\
\hline Infección Urinaria & 3.7 & 3.0 & 310 & 210 & $3 \mathrm{~N}$ \\
\hline Infección Urinaria & 1.4 & 0.7 & 174 & 48 & $3 \mathrm{~N}$ \\
\hline $\begin{array}{l}\text { Infección Urinaria } \\
\text { Colecistitis }\end{array}$ & 2.8 & 1.4 & 200 & 200 & $2 \mathrm{~N}$ \\
\hline $\begin{array}{l}\text { Calculosa } \\
\text { Colecistitis }\end{array}$ & 1.9 & 1.1 & 140 & 150 & $2 \mathrm{~N}$ \\
\hline $\begin{array}{l}\text { Calculosa } \\
\text { Colecistitis }\end{array}$ & 1.0 & 0.6 & 64 & 66 & $\mathrm{~N}$ \\
\hline $\begin{array}{l}\text { Colecistitis } \\
\text { Calculosa } \\
\text { Colecistitis }\end{array}$ & 1.0 & 0.4 & 70 & 68 & $\mathrm{~N}$ \\
\hline $\begin{array}{l}\text { Calculosa } \\
\text { Colecistitis }\end{array}$ & 1.2 & 0.6 & 220 & 200 & $\mathrm{~N}$ \\
\hline $\begin{array}{l}\text { Calculosa } \\
\text { Fiebre de causa }\end{array}$ & 2.2 & 1.1 & 20 & 15 & $\mathrm{~N}$ \\
\hline $\begin{array}{l}\text { Indet. } \\
\text { Fiebre de causa }\end{array}$ & 1.6 & 1.8 & 111 & 90 & $3 \mathrm{~N}$ \\
\hline $\begin{array}{l}\text { Indet. } \\
\text { Fiebre de causa }\end{array}$ & 1.2 & 0.7 & 80 & 30 & $2 \mathrm{~N}$ \\
\hline $\begin{array}{l}\text { Indet. } \\
\text { Obstrucción }\end{array}$ & 1.7 & 1.1 & 176 & 111 & $2 \mathrm{~N}$ \\
\hline $\begin{array}{l}\text { Intestinal } \\
\text { Obstrucción }\end{array}$ & 3.1 & 2.3 & 28 & 23 & $3 \mathrm{~N}$ \\
\hline Intestinal & 3.0 & 2.0 & 740 & 740 & $2 \mathrm{~N}$ \\
\hline $\begin{array}{l}\text { Absceso Cerebral } \\
\text { Gastritis Erosiva }\end{array}$ & 0.8 & 0.4 & 54 & 38 & $\mathrm{~N}$ \\
\hline $\begin{array}{l}\text { - HDA } \\
\text { Absceso }\end{array}$ & 1.7 & 0.3 & 940 & 640 & $\mathrm{~N}$ \\
\hline Apendicular Resi & 4.4 & 2.4 & 26 & 10 & $2 \mathrm{~N}$ \\
\hline Bronconeumonía & 2.8 & 2.0 & 200 & 200 & $2 \mathrm{~N}$ \\
\hline TBC Pulmonar & 1.41 & 0.8 & 16 & 11 & $\mathrm{~N}$ \\
\hline
\end{tabular}


Las manifestaciones clínicas predominantes son las de la enfermedad de fondo; pero se encuentran fiebre, coluria, hepatomegalia e ictericia en los veinte pacientes.

\section{DISCUSION}

Ante una injuria leve del hígado es probable que lo primero que se modifique sea la función celular sin cambios anatómicos evidenciales por los medios actualmente disponibles.

Así, por ejemplo, es posible que inicialmente ocurra un cambio en la permeabilidad de la membrana celular que permite la salida de elementos endocelulares como las transaminasas y su consecuente elevación en la sangre o se presenten modificaciones a nivel del polo biliar del hepatocito que afectan su excreción. Esto corresponde a los casos señalados por Levine (2), que nosotros entendemos como disfunción hepática secundaria a otros procesos (3) y que revelaría un cierto grado de sufrimiento del hígado con o sin manifestaciones clínicas objetivas.

Cuando la perturbación es mayor, se producen cambios anatómicos. En los casos de una reacción inflamatoria hay una gama de posibilidades que van desde el edema, la infiltración celular, hasta la necrosis del parénquima hepático en grado variable. Esto es lo que se designa con el nombre de hepatitis.

Los morfólogos pueden reconocer la presencia de una hepatitis. Si los cambios son característicos se habla de una hepatitis específica; así es posible identificar la hepatitis viral, la séptica, algunas granulomatosas, las relacionadas con ciertas drogas, etc.

En otros casos las alteraciones inflamatorias producidas en el hígado no son susceptibles de atribuirse a causas hepato agresivas bien definidas, por lo que se les designa como hepatitis inespecífica.

Los cambios histológicos ofrecen gran variedad: hay áreas de necrosis focal que comprometen hepatocitos aislados o pequeños grupos de células, a veces con cuerpos acidófilos o degeneración hidrópica. Puede haber discreta esteatosis o leve colestasis. En algunos otros casos hay infiltración en los espacios porta por macrófagos, linfocitos y neutrófilos. Este aspecto puede semejar una hepatitis crónica persistente de grado leve; pero la historia clínica y la evolución son completamente diferentes (4).

Se la describe en los textos de patología recibiendo diversas denominaciones. Ruebnes y Montgomery (5) la mencionan como necrosis focal no específica. Hay quienes la designan como hepatitis infiltrativa (6), en tanto que Popper y Shaffner (7) la nominan

hepatitis reactiva inespecífica o hepatitis reaccional, término generalizado más no siempre aceptado, pues todas las hepatitis son consecuencia de una reacción al proceso inflamatorio.

L. Frederik Fenstr (8) propuso la denominación de hepatopatía reactiva y reporta un caso en el que luego de múltiples estudios se llega incluso a una laparotomía exploratoria, confirmándose la presencia de una diverticulitis sigmoidea, remitiendo el cuadro totalmente luego de la ablación del sigmoides comprometido. 
En el grupo estudiado por nosotros en el Hospital Cayetano Heredia predominó el sexo femenino en la proporción de 3 a 1 y el $60 \%$ de los casos fueron menores de 30 años, distribuyéndose con igual frecuencia en cada una de las tres primeras décadas de la vida.

En todos estos enfermos se realizó biopsia hepática y los estudios anatomopatológico revelaron alteraciones variables con leve injuria focal, infiltración celular de tipo portal, cambios mínimos en los colangiolos y en los sinusoides. Es lo que tipifica a una hepatitis inespecífica.

Nuestra casuística incluye a pacientes con diferentes diagnósticos, siendo los más frecuentes infección urinaria $25 \%$, colecistitis calculosa $25 \%$ y fiebre de causa indeterminada $15 \%$.

Clínicamente predominó el cuadro de la enfermedad de fondo, que en algún momento de su evolución se complicó con coluria, ictericia de grado variable y usualmente modificación de la curva febril, que reapareció en unos o se hizo más acentuada o prolongada en otros. En el examen físico, además de la ictericia y la fiebre, en todos los casos se evidenció hepatomegalia de tamaño variable, pero nunca muy grande, algo dolorosa.

Los exámenes de laboratorio confirmaron el daño hepático, aun cuando no siempre se pudieron realizar en el momento de mayor evidencia del cuadro. Lo más destacable en el grupo investigado fue:hiperbilirrubinemia a predominio de la conjugada en todos los casos, en el $80 \%$ la bilirrubina total alcanzó niveles que no sobrepasaron los $3 \mathrm{mgrs} \%$, aunque existieron rangos mayores. Hubo leve elevación de transaminasas, sin franco predominio de ninguna de ellas y en el $85 \%$ no fueron mayores de 250 unidades, sin embargo se presentaron algunos con cifras superiores, en el $60 \%$ la fosfatasa alcalina estuvo elevada en 2 a 3 veces no normal.

La hiperbilirrubinemia no muy acentuada, a predominio de la conjugada, con discreta elevación de las transaminasas y la fosfatasa alcalina en 2 a 3 veces lo normal, fueron los hallazgos más constantes en los casos evaluados

Creemos que la presencia de esta hepatitis inespecífica asociada a otra patología es la consecuencia de la acción de uno o varios factores sobre un hígado susceptible en un paciente gravemente enfermo. Señalamos entre los más importantes:

- Ciertos procesos tóxico-metabólicos como las atribuidas endotoxinas bacterianas presentes en los procesos sépticos que ocurren en la mayoría de estos enfermos y que han sido señalados en la literatura $(9,10)$.

- Alteraciones circulatorias que perturban la irrigación la irrigación hepática.

- Procesos inmunológicos capaces de dañar al hígado.

- Otros aún no conocidos.

Fenster (8) está de acuerdo en la casualidad multifactorial y en la importancia de la sepsis, señalando además la presencia de ciertas sustancias humorales secretadas por algunas neoplasias extrahepáticas, la disminución de la nutrición peroral con la consecuente reducción de la secreción de sales biliares, la hipovolemia con hipoxia, el estrés metabólico y la sobrecarga de bilirrubina por hemólisis o transfusiones. 
El pronóstico está siempre relacionado a la enfermedad de fondo y el tratamiento debe ser correspondiente, evitando en lo posible los hepatotóxicos.

\section{BIBLIOGRAFIA}

1.Leevy CM, Popper H, Sherlock S. Diseases of the Liver and Biliary Tract: Standarization of Nomenclature, Diagnostic Criteria and Diagnostic Methodology: United States 1976. Fogarty International Center Proceeding $\mathrm{N}^{\circ}$ 22.DHEW publication num (NIH) 76-725.

2.Levine RA and Klatskin G. Unconjugated hyperbilirrubinemia in the absence of overt hemolysis. Importance of acquired disease as an etiologic factor in 366 adolescent and adult subjects. Am J Med 1964; 36: 541-552.

3.Berrios J. Clasificación de las Ictericias. Diagnóstico 1978; 2: 26-29.

4.Gerber MA and Thung SN. Viral hepatitis: Pathology en Berk JE Ed. Bockus Gastroenterology. W.B. Saunders Company. Philadelphia 1985, pp: 2825-2855.

5.Ruebner HB and Montgomery KC. Pathology of the Liver and Biliary Tract. John Wiley \& Sons. New York 1982,60.

6.Mateer JG, Hartman FW. Balz JI Faelis LD, McGraw AB and Steele HH. Combined liver biopsy and liver function study in 132 cases of cholelitiasis and 31 cases of peptic ulcer. Gastroenterology 1948; 11: 284-302.

7.Popper H. and Schaffner F. El Hígado. Su Estructura y Funcion Ed. Noguer S.A. Barcelona 1962. p: 483.

8.Fenster LF. Reactive Hepatopathy. Liver signs without primary liver disease. Postgrad Med 1984; 76:62-65.

9.Zimmerman HJ and Thomas LJ. Liver in pneumococcal pneumonia. Observations in 94 cases on liver function and jaundice in pneumonia. J Lab Clin Med 1950; 35: 556-567.

10.Kleckner MS; Stauffer MH, Bargen JA, and Dockerty MB. Hepatic lesions in living patient with chronic ulcerative colitis as demonstrated by needle biopsy. Gastroenterology 1952; 22:13-33. 